\title{
Galerkin finite element approximation of general linear second order hyperbolic equations
}

\author{
M. Basson* \& N. F. J. van Rensburg ${ }^{\dagger}$ \\ Department of Mathematics and Applied Mathematics, University of Pretoria, South Africa
}

\begin{abstract}
In this article we derive error estimates for the Galerkin approximation of a general linear second order hyperbolic partial differential equation. The results can be applied to a variety of cases e.g. vibrating systems of linked elastic bodies. The results generalize the work of Baker [1] and also allow for viscous type damping. Splitting the proofs for the semidiscrete and fully discrete cases not only simplifies the proofs but less restrictive regularity assumptions are required.
\end{abstract}

\section{Introduction}

In this paper we consider the Galerkin finite element approximation of a solution of a general second order hyperbolic equation. This includes the general second order wave equation but also vibrating beams, plates etc., since the variational forms are the same as for the wave equation. Even systems of linked elastic bodies can be accommodated, see Section 7 . There is a restriction to the theory however and this relates to damping. Damping at the boundary or interfaces is for example not allowed.

\footnotetext{
${ }^{*}$ Corresponding author. Email: madelein.basson@up.ac.za
}

${ }^{\dagger}$ Email: nic.vanrensburg@up.ac.za 
The general second order hyperbolic partial differential equation is formulated in Section 2 in variational form and is referred to as Problem G. Conditions for the existence of a unique solution are given.

Convergence of the Galerkin approximation for the wave equation was considered in the book [2] and article [3] both published in 1973. In [2] a sketch of a proof of convergence for the undamped case is presented. In [3] error estimates are derived for the undamped case and a problem with boundary damping. These error estimates are for the semi discrete approximation and the fully discrete approximation.

Dupont [3] made the assumption that the exact solution has a second order time derivative with respect to the energy norm, which is rather restrictive. In 1976 Baker derived error estimates with "minimal smoothness requirements on the solution", but no damping [1]. This article had a significant impact on the literature and other authors adapted his methods or were influenced by it, see e.g. [4], [5], [6], [7] and [8].

We generalize the results of Baker [1]. The spaces $\mathcal{L}^{2}(\Omega)$ and $H_{0}^{1}(\Omega)$ are replaced by general Hilbert spaces $W$ and $V$, where $V$ is a subset of $W$. In the general problem the $\mathcal{L}^{2}$-norm is replaced by the general inertia norm $\|\cdot\|_{W}$ and the well known energy norm for the wave equation (equivalent to the $H^{1}$ norm) is replaced by a general energy norm $\|\cdot\|_{V}$. Naturally certain properties of the spaces and their norms need to be assumed in a general setting (see Section 2). An attempt to obtain error estimates for a general hyperbolic problem was made in [9] and [10]. The assumptions made were also rather restrictive. The differentiability assumption is the same as in [3] and modal damping is assumed.

As mentioned, Problem $\mathrm{G}$ in Section 2 is the abstract variational problem in Hilbert space. (The problem in [1] is a special case.) So called viscous type damping is included. It is natural to ask whether this restriction is necessary. We believe that boundary or interface damping requires a different approach based on the fact that existence results are influenced by the type of damping.

The existence result in Section 2 is presented in variational form. This is more convenient for the finite element method as one can compare conditions for existence to conditions for convergence. We see that sufficient conditions for existence are also used to prove convergence but they seem not to be sufficient and more regularity of solutions is assumed.

This article is not merely about generalization but other contributions to 
the theory are also made. Consider for example the significance of the semidiscrete approximation. In [3], [1] and other publications (e.g. [5], [6] and [7]) error estimates are derived for the semi discrete approximation and then for the fully discrete approximation without using the results already obtained. In some cases the semi-discrete approximation is ignored. We derive an error estimate for the semi-discrete approximation with respect to the inertia norm and then an estimate for the error in approximating the solution of the semidiscrete problem by the fully discrete approximation with respect to the same norm. The final estimate follows trivially by the triangle inequality. This approach has two advantages. It is not necessary to assume the existence of a third or fourth order derivative for the exact solution and the convergence analysis for the fully discrete approximation is simplified.

In [1] error estimates are derived "using $L^{2}$-projections of the initial data as starting values". The fact that the starting values for the approximation are a projection, is used to derive estimates (see Section 4). But it is not always possible to determine the projections exactly. It is not clear in such an event how the error estimates can be interpreted. In our approach projections are not used as initial approximations in the fundamental estimate. The errors for the initial approximations are dealt with at a later stage (see Section 5).

It is well known that the smoothness of a solution is important in convergence analysis. In this paper we attempt to pinpoint exactly where the regularity in space and time is used in the analysis. Regarding time, see Sections 4 and 6 and for spatial regularity, see Section 5. An issue that deserves attention here is the differentiability of the projection of the exact solution. It is possible that the exact solution does not have a second order derivative with respect to the energy norm but its projection may have a second order derivative (see Section 3).

To summarize, error estimates for the semi-discrete approximation are presented in Section 5 and those for the fully discrete appear in Section 6 . Finally, applications are discussed in Section 7.

Remark To compare results in different articles is time consuming due to differences in notation. We have used the notation from [2]. 


\section{Existence and uniqueness of solutions}

In this section we consider the general second order hyperbolic equation or general linear vibration problem in variational form. Let $X, W$ and $V$ denote Hilbert spaces such that $V \subset W \subset X$.

\section{Notation}

$X$ has inner product $(\cdot, \cdot)_{X}$ and norm $\|\cdot\|_{X}$.

$W$ has inner product $c$ and norm $\|\cdot\|_{W}$.

$V$ has inner product $b$ and norm $\|\cdot\|_{V}$.

Let $J$ be a bounded or unbounded interval, either an open interval containing zero or of the form $[0, \tau)$ or $[0, \infty)$. Let $Y$ be any Hilbert space and consider a function $u$ on $J$ with values in $Y$.

We write $u^{\prime}(t) \in Y$ if the derivative exists with respect to the norm of $Y$;

$$
\begin{aligned}
& u^{(k)} \in \mathcal{L}^{2}(J ; Y) \text { if } u^{(k)}(t) \in Y \text { for each } t \text { and } \int_{J}\left\|u^{(k)}\right\|_{Y}^{2}<\infty ; \\
& u \in C^{k}(J ; Y) \text { if } u^{(k)} \in C(J ; Y) .
\end{aligned}
$$

Consider a bilinear form $a$ defined on $W$ and the following general problem.

\section{Problem G}

Given a function $f: J \rightarrow X$, determine a function $u \in C(J ; V)$ such that $u^{\prime}$ is continuous at 0 and for each $t \in J$

$$
\begin{gathered}
u(t) \in V, \quad u^{\prime}(t) \in V, \quad u^{\prime \prime}(t) \in W \\
c\left(u^{\prime \prime}(t), v\right)+a\left(u^{\prime}(t), v\right)+b(u(t), v)=(f(t), v)_{X} \quad \text { for each } v \in V, \\
\text { while } u(0)=u_{0}, \quad u^{\prime}(0)=u_{1} .
\end{gathered}
$$

Assumptions The following assumptions are used for existence theory as well as convergence theory.

E1 $V$ is dense in $W$, 
E2 There exists a constant $C_{b}$ such that $\|v\|_{W} \leq C_{b}\|v\|_{V}$ for each $v \in V$,

E3 There exists a constant $C_{c}$ such that $\|v\|_{X} \leq C_{c}\|v\|_{W}$ for each $v \in W$,

E4 The bilinear form $a$ is nonnegative, symmetric and bounded on $W$, i.e.

$$
|a(u, v)| \leq K_{a}\|u\|_{W}\|v\|_{W} .
$$

If the bilinear form $a$ satisfies Assumption E4 we refer to the damping as viscous type since it is valid for viscous damping in the wave equation and other vibration problems. (In [11] it is referred to as weak damping.) The assumption is not valid for boundary damping; it does not hold for the model in [3].

Remark In the rest of this paper, except Section 7, $b$ and $c$ are inner products for the spaces $V$ and $W$ respectively and $a$ is a bilinear form satisfying assumption A4.

For the wave equation $\|\cdot\|_{X}$ is just the $\mathcal{L}^{2}$-norm and in many applications the norms $\|\cdot\|_{X}$ and $\|\cdot\|_{W}$ are equivalent.

\section{Definition}

$E_{b}=\{x \in V \mid$ there exists a $y \in W$ such that $c(y, v)=b(x, v)$ for all $v \in V\}$.

Theorem 2.1. Suppose Assumptions E1, E2, E3 and E4 hold, then there exists a unique solution

$$
u \in C^{1}(J ; V) \cap C^{2}(J ; W),
$$

for Problem $G$ for each $u_{0} \in E_{b}, u_{1} \in V$ and each $f \in C^{1}(J ; X)$. If $f=0$ then $u \in C^{1}((-\infty, \infty) ; V) \cap C^{2}((-\infty, \infty) ; W)$.

Proof. See [11].

Problem $\mathrm{G}$ is equivalent to a first order differential equation in the product space $V \times W: y^{\prime}=A y$ with $y(0)=\left\langle u_{0}, u_{1}\right\rangle$. The operator $A$ is determined by the three bilinear forms $c, b$ and $a$ and $\mathcal{D}(A)=E_{b} \times V$. The construction of $A$ is given in [11]. Due to Assumptions E1, E2 and E4, $A$ is the infinitesimal generator of a $C_{0}$ group of contractions.

\section{Remarks}


1. The definition of $E_{b}$ implies that $E_{b}$ is the set of equilibrium states.

2. Existence results may be found in other publications e.g. [12, 1972] and $[13,1977]$ but the result in $[11,2002]$ is convenient for this paper.

\section{Galerkin approximation}

In this section we consider the Galerkin approximation. Assumptions E1, E2, E3 and E4 are assumed to hold for the general convergence theory. Suppose $S^{h}$ is a finite dimensional subspace of $V$. (The symbol $h$ has no meaning at this stage, but it is customary to use the notation.)

\section{Problem $G^{h}$}

Given a function $f: J \rightarrow X$, find a function $u_{h} \in C^{2}(J)$ such that for each $t \in J$

$$
\begin{gathered}
c\left(u_{h}^{\prime \prime}(t), v\right)+a\left(u_{h}^{\prime}(t), v\right)+b\left(u_{h}(t), v\right)=(f(t), v)_{X} \quad \text { for each } v \in S^{h}, \\
\text { while } \quad u_{h}(0)=u_{0}^{h}, \quad u_{h}^{\prime}(0)=u_{1}^{h} .
\end{gathered}
$$

The initial values $u_{0}^{h}$ and $u_{1}^{h}$ are elements of $S^{h}$ as close as possible to $u_{0}$ and $u_{1}$, see Section 5 .

Since $c$ is an inner product and $S^{h}$ is finite dimensional, existence is no problem if $f$ is continuous (see Section 6).

Theorem 3.1. If $f \in C(J, X)$, then there exists a unique solution $u_{h} \in$ $C^{2}(J)$ for Problem $G^{h}$ for each $u_{0}^{h}$ and $u_{1}^{h}$ in $S^{h}$. If $f=0$ then $u_{h} \in$ $C^{2}((-\infty, \infty))$.

To find an estimate for the discretization error $e_{h}(t)=u(t)-u_{h}(t)$, a projection is used. The projection operator $P_{h}$ is defined by

$$
b\left(u-P_{h} u, v\right)=0 \text { for each } v \in S^{h} .
$$

If no confusion is possible we will write $P$ for $P_{h}$. We also use the symbol $P$ to denote the projection $P u$ of a function $u$, i.e. $(P u)(t)=P u(t)$ for each $t \in J$. Using the projection we split the error. Let $e(t)=P u(t)-u_{h}(t)$ and $e_{p}(t)=u(t)-P u(t)$. Then

$$
e_{h}(t)=e_{p}(t)+e(t)
$$


Estimates for the norm of $e_{p}(t)$ are obtained from interpolation theory in applications (see Sections 5 and 7). The problem is to determine an estimate for $e(t)$, the difference between the projection $P u(t)$ and the Galerkin approximation $u_{h}(t)$. Our aim is to estimate this error in terms of the projection error and errors for the initial conditions.

The convergence results that can be obtained, depend on the properties of the solution. But these may vary from problem to problem. The weakest assumption is the result of the existence theorem: if $u$ is a solution of Problem $\mathrm{G}$, then $u \in C^{1}(J ; V) \cap C^{2}(J ; W)$.

Lemma 3.1. If $u \in C^{1}(J, V)$, then $P u \in C^{1}(J)$ and $(P u)^{\prime}(t)=P u^{\prime}(t)$.

Proof. As the projection operator $P$ is a bounded linear operator with norm less than one, we have that

$\left\|(\delta t)^{-1}(P u(t+\delta t)-P u(t))-P u^{\prime}(t)\right\|_{V} \leq\left\|(\delta t)^{-1}(u(t+\delta t)-u(t))-u^{\prime}(t)\right\|_{V}$.

Clearly $P u \in C^{1}(J, V)$ and $(P u)^{\prime}(t)=P u^{\prime}(t)$.

We need the following property of the solution for the convergence analysis.

\section{Assumption A1}

The solution $u \in C(J, V)$ of Problem $\mathrm{G}$ has the property that $(P u) \in C^{2}(J)$.

Remark In [3] the assumption $u \in C^{2}(J, V)$ is used and it is then possible to prove that $P u \in C^{2}(J)$. Baker [1] derived error estimates for the undamped case using a weaker assumption, but assumed A1 without explicitly mentioning it. The assumption in [3] is sufficient to prove A1 but not necessary.

It is important to note that we use the fact that $(P u)^{\prime}=P u^{\prime}$ but not $(P u)^{\prime \prime}=$ $P u^{\prime \prime}$.

If the solution $u$ of Problem $\mathrm{G}$ satisfies Assumption A1, then $e_{p} \in C^{2}(J, W)$. This is easy to see since $e_{p}=u-P u$ and since norms in $S^{h}$ are equivalent, $P u \in C^{2}(J, W)$.

Proposition 3.1. If the solution $u$ of Problem G satisfies Assumption A1, then

$$
c\left(e_{h}^{\prime \prime}(t), v\right)+a\left(e_{h}^{\prime}(t), v\right)+b(e(t), v)=0 \text { for all } v \in S^{h} .
$$


Proof. By subtracting (3.1) from (2.1) we obtain the following identity. For any $v \in S^{h}$,

$$
c\left(u^{\prime \prime}(t)-u_{h}^{\prime \prime}(t), v\right)+a\left(u^{\prime}(t)-u_{h}^{\prime}(t), v\right)+b\left(u(t)-u_{h}(t), v\right)=0 .
$$

Add and subtract $P u$ in the bilinear form $b$ above and we have

$$
c\left(e_{h}^{\prime \prime}(t), v\right)+a\left(e_{h}^{\prime}(t), v\right)+b(u(t)-P u(t), v)+b\left(P u(t)-u_{h}(t), v\right)=0,
$$

for all $v \in S^{h}$. Since $P$ is a projection with respect to the inner product $b$, the result follows.

\section{Fundamental estimate}

The following lemma is a generalization of an estimate for $e=P u-u_{h}$ in [1] and we also include damping.

Lemma 4.1. If the solution $u$ of Problem G satisfies Assumption A1, then for $t \in[0, T]$,

$$
\begin{aligned}
\|e(t)\|_{W} \leq & \sqrt{2}\left(\|e(0)\|_{W}+3 T\left\|e_{h}^{\prime}(0)\right\|_{W}+3 \int_{0}^{T}\left\|e_{p}^{\prime}(t)\right\|_{W}\right. \\
& \left.+3 K_{a} T\left\|e_{h}(0)\right\|_{W}+3 K_{a} \int_{0}^{T}\left\|e_{p}(t)\right\|_{W}\right) .
\end{aligned}
$$

Proof. In Proposition 3.1 we have

$$
c\left(e_{h}^{\prime \prime}(t), v\right)+a\left(e_{h}^{\prime}(t), v\right)+b(e(t), v)=0 \text { for all } v \in S^{h} .
$$

Now, if $v$ is a function on $[0, T]$ with values in $S^{h}$, then

$$
c\left(e_{h}^{\prime \prime}(t), v(t)\right)+a\left(e_{h}^{\prime}(t), v(t)\right)+b(e(t), v(t))=0 .
$$

Let $v^{\prime}(t)=e(t)$, then

$$
\begin{aligned}
\frac{d}{d t}[ & \left.\frac{1}{2} c(e, e)-\frac{1}{2} b(v, v)-c\left(e_{h}^{\prime}, v\right)-a\left(e_{h}, v\right)\right] \\
& =c\left(e^{\prime}, e\right)-b(e, v)-c\left(e_{h}^{\prime \prime}, v\right)-a\left(e_{h}^{\prime}, v\right)-c\left(e_{h}^{\prime}, e\right)-a\left(e_{h}, e\right) \\
& =-c\left(e_{p}^{\prime}, e\right)-a\left(e_{h}, e\right)
\end{aligned}
$$

from (4.1). 
Choose $v(\tau)=0$ for some $\tau \in(0, T)$, and integrate (4.2) between 0 and $\tau$. We obtain

$$
\begin{gathered}
-\left[\frac{1}{2} c(e(0), e(0))-\frac{1}{2} b(v(0), v(0))-c\left(e_{h}^{\prime}(0), v(0)\right)-a\left(e_{h}(0), v(0)\right)\right] \\
+\frac{1}{2} c(e(\tau), e(\tau))=-\int_{0}^{\tau} c\left(e_{p}^{\prime}, e\right)-\int_{0}^{\tau} a\left(e_{p}, e\right)-\int_{0}^{\tau} a(e, e) .
\end{gathered}
$$

Since $a(e, e) \geq 0$ and $b(v(0), v(0)) \geq 0$, we have

$$
\begin{gathered}
\frac{1}{2} c(e(\tau), e(\tau)) \leq \frac{1}{2} c(e(0), e(0))-c\left(e_{h}^{\prime}(0), v(0)\right)-a\left(e_{h}(0), v(0)\right) \\
-\int_{0}^{\tau} c\left(e_{p}^{\prime}, e\right)-\int_{0}^{\tau} a\left(e_{p}, e\right)
\end{gathered}
$$

and consequently

$$
\begin{gathered}
c(e(\tau), e(\tau)) \leq c(e(0), e(0))+2 \int_{0}^{\tau} c\left(e_{h}^{\prime}(0), e\right)-2 \int_{0}^{\tau} c\left(e_{p}^{\prime}, e\right) \\
+2 \int_{0}^{\tau} a\left(e_{h}(0), e\right)-2 \int_{0}^{\tau} a\left(e_{p}, e\right) .
\end{gathered}
$$

Estimates for the terms on the right are now required. We use Young's inequality (see e.g. [14]) and denote $\max _{t \in[0, T]}$ by max.

$$
\begin{aligned}
\left|2 \int_{0}^{\tau} c\left(e_{h}^{\prime}(0), e\right)\right| & \leq 2 \int_{0}^{\tau}\left|c\left(e_{h}^{\prime}(0), e\right)\right| \\
& \leq 2 \int_{0}^{\tau}\left\|e_{h}^{\prime}(0)\right\|_{W}\|e\|_{W} \\
& \leq 2 T \max \|e(t)\|_{W}\left\|e_{h}^{\prime}(0)\right\|_{W} \\
& \leq \frac{1}{9}\left[\max \|e(t)\|_{W}\right]^{2}+9 T^{2}\left\|e_{h}^{\prime}(0)\right\|_{W}^{2} .
\end{aligned}
$$

Similarly

$$
\begin{aligned}
\left|2 \int_{0}^{\tau} a\left(e_{h}(0), e\right)\right| & \leq 2 K_{a} T \max \|e(t)\|_{W}\left\|e_{h}(0)\right\|_{W} \\
& \leq \frac{1}{9}\left[\max \|e(t)\|_{W}\right]^{2}+9 K_{a}{ }^{2} T^{2}\left\|e_{h}(0)\right\|_{W}^{2}
\end{aligned}
$$

also using E4. Next

$$
\begin{aligned}
\left|2 \int_{0}^{\tau} c\left(e_{p}^{\prime}, e\right)\right| & \leq 2 \max \|e(t)\|_{W} \int_{0}^{\tau}\left\|e_{p}^{\prime}\right\|_{W} \\
& \leq \frac{1}{9}\left[\max \|e(t)\|_{W}\right]^{2}+\left(3 \int_{0}^{\tau}\left\|e_{p}^{\prime}\right\|_{W}\right)^{2}
\end{aligned}
$$


and

$$
\begin{aligned}
\left|2 \int_{0}^{\tau} a\left(e_{p}, e\right)\right| & \leq 2 K_{a} \max \|e(t)\|_{W} \int_{0}^{\tau}\left\|e_{p}\right\|_{W} \\
& \leq \frac{1}{9}\left[\max \|e(t)\|_{W}\right]^{2}+\left(3 K_{a} \int_{0}^{\tau}\left\|e_{p}\right\|_{W}\right)^{2} .
\end{aligned}
$$

Using these estimates in (4.4), we obtain

$$
\begin{aligned}
\|e(\tau)\|_{W}^{2} \leq \frac{4}{9} & \max \|e(t)\|_{W}^{2}+\|e(0)\|_{W}^{2}+9 T^{2}\left\|e_{h}^{\prime}(0)\right\|_{W}^{2}+9 \int_{0}^{T}\left\|e_{p}^{\prime}(t)\right\|_{W}^{2} \\
+ & 9 K_{a}^{2} T^{2}\left\|e_{h}(0)\right\|_{W}^{2}+9 K_{a}^{2} \int_{0}^{T}\left\|e_{p}(t)\right\|_{W}^{2}
\end{aligned}
$$

Since this is true for all $\tau \in(0, T)$, we have that

$$
\begin{aligned}
\max \|e(t)\|_{W}^{2} \leq \frac{1}{2} & \max \|e(t)\|_{W}^{2}+\|e(0)\|_{W}^{2}+9 T^{2}\left\|e_{h}^{\prime}(0)\right\|_{W}^{2}+9 \int_{0}^{T}\left\|e_{p}^{\prime}(t)\right\|_{W}^{2} \\
& +9 K_{a}^{2} T^{2}\left\|e_{h}(0)\right\|_{W}^{2}+9 K_{a}^{2} \int_{0}^{T}\left\|e_{p}(t)\right\|_{W}^{2},
\end{aligned}
$$

and hence

$$
\begin{gathered}
\max \|e(t)\|_{W}^{2} \leq 2\left(\|e(0)\|_{W}^{2}+9 T^{2}\left\|e_{h}^{\prime}(0)\right\|_{W}^{2}+9 \int_{0}^{T}\left\|e_{p}^{\prime}(t)\right\|_{W}^{2}+9 K_{a}^{2} T^{2}\left\|e_{h}(0)\right\|_{W}^{2}\right. \\
\left.\quad+9 K_{a}^{2} \int_{0}^{T}\left\|e_{p}(t)\right\|_{W}^{2}\right) \\
\leq 2\left(\|e(0)\|_{W}+3 T\left\|e_{h}^{\prime}(0)\right\|_{W}+3 \int_{0}^{T}\left\|e_{p}^{\prime}(t)\right\|_{W}+3 K_{a} T\left\|e_{h}(0)\right\|_{W}\right. \\
\left.+3 K_{a} \int_{0}^{T}\left\|e_{p}(t)\right\|_{W}\right)^{2} .
\end{gathered}
$$

Suppose that there is no damping, i.e. $a=0$ in (4.4). Then, using the same arguments as in the proof above, we have the following corollary.

Corollary 4.1. If the solution $u$ of Problem $G$ with $a=0$ satisfies Assumption $A 1$, then for $t \in[0, T]$,

$$
\|e(t)\|_{W} \leq \sqrt{2}\|e(0)\|_{W}+2 T\left\|e_{h}^{\prime}(0)\right\|_{W}+4 \sqrt{T} \max _{t \in[0, T]}\left\|e_{p}^{\prime}(t)\right\|_{W}
$$


Now, consider the undamped case with the additional assumption that $c\left(u_{1}-u_{1}^{h}, v(0)\right)=c\left(e_{h}^{\prime}(0), v(0)\right)=0$. The estimate in (4.3) reduces to

$$
\frac{1}{2} c(e(\tau), e(\tau)) \leq \frac{1}{2} c(e(0), e(0))-\int_{0}^{\tau} c\left(e_{p}^{\prime}, e\right)
$$

and we have the following result.

Corollary 4.2. Suppose the solution $u$ of Problem $G$ with $a=0$ satisfies Assumption A1. If $c\left(u_{1}-u_{1}^{h}, v\right)=0$ for all $v \in S^{h}$, then for $t \in[0, T]$,

$$
\|e(t)\|_{W} \leq \sqrt{2}\|e(0)\|_{W}+2 \sqrt{T} \max _{t \in[0, T]}\left\|e_{p}^{\prime}(t)\right\|_{W} .
$$

This is a generalization of the estimate for $e=P u-u_{h}$ in (3.13) in [1]. However, it is not necessary to make the assumption at this stage.

\section{Convergence and error estimates for the semi-discrete approximation}

The error estimates follow readily from Lemma 4.1.

Theorem 5.1. If the solution u of Problem G satisfies Assumption A1, then

$$
\begin{aligned}
& \left\|u(t)-u_{h}(t)\right\|_{W} \leq\left\|e_{p}(t)\right\|_{W}+\sqrt{2}\left(\left\|P u_{0}-u_{0}\right\|_{W}+3 T\left\|u_{1}-u_{1}^{h}\right\|_{W}+3 \int_{0}^{T}\left\|e_{p}^{\prime}(t)\right\|_{W}\right. \\
& \left.+\left(1+3 K_{a} T\right)\left\|u_{0}-u_{0}^{h}\right\|_{W}+3 K_{a} \int_{0}^{T}\left\|e_{p}(t)\right\|_{W}\right)
\end{aligned}
$$

for each $t \in[0, T]$.

Proof. From (3.2), $\left\|u(t)-u_{h}(t)\right\|_{W} \leq\left\|e_{p}(t)\right\|_{W}+\|e(t)\|_{W}$. The result follows from Lemma 4.1 since $\left\|P u_{0}-u_{0}^{h}\right\|_{W} \leq\left\|P u_{0}-u_{0}\right\|+\left\|u_{0}-u_{0}^{h}\right\|$.

In applications it is necessary to consider the errors on the right hand side of the estimate (5.1).

To formulate an assumption regarding the error when an element of $V$ is approximated by an element of $S^{h}$, we suppose $h$ is a parameter related to the dimension $n$ of $S^{h}$ and $h \rightarrow 0$ as $n \rightarrow \infty$. 


\section{Assumption A2}

There exists a subspace $H$ of $V$ and a positive integer $\alpha$ such that if $w \in H$, then

$$
\inf _{v \in S^{h}}\|w-v\|_{V} \leq \widehat{C} h^{\alpha} \mid\|w\|_{H},
$$

where $\||w|\|_{H}$ is a norm or semi-norm for $H$.

Using Assumption A2, estimates for the projection errors in (5.1) can be obtained. For example, $\left\|e_{p}^{\prime}(t)\right\|_{W}=\left\|u^{\prime}(t)-P u^{\prime}(t)\right\|_{W} \leq C_{b}\left\|u^{\prime}(t)-P u^{\prime}(t)\right\|_{V}$ by Assumption E2 and Lemma 3.1. Therefore

$$
\left\|e_{p}^{\prime}(t)\right\|_{W} \leq C_{b} \widehat{C} h^{\alpha}\left|\left\|u^{\prime}(t) \mid\right\|_{H}\right.
$$

If we now choose $u_{0}^{h}=P u_{0}$ and $u_{1}^{h}=P u_{1}$ as in [1], then we have for example

$$
\left\|u_{0}-u_{0}^{h}\right\|_{W} \leq C_{b} \widehat{C} h^{\alpha}|| \mid u_{0} \|_{H},
$$

and we are done. This is in general not possible. However, the estimate in Assumption A2 is derived from the interpolation error in applications. If we choose $u_{0}^{h}=\Pi u_{0}$ and $u_{1}^{h}=\Pi u_{1}$, where $\Pi$ denotes the interpolation operator, then we have the same estimate as in (5.2).

Theorem 5.2. Suppose Assumption A2 holds and $u_{0}^{h}=\Pi u_{0}$ and $u_{1}^{h}=\Pi u_{1}$. If the solution $u$ of Problem $G$ satisfies Assumption A1, $u(t) \in H$ and $u^{\prime}(t) \in H$, then

$$
\begin{aligned}
\left\|u(t)-u_{h}(t)\right\|_{W} \leq & C_{b} \widehat{C} h^{\alpha}\|\mid u(t)\| \|_{H}+\sqrt{2} C_{b} \widehat{C} h^{\alpha}\left(3 T \max \left\|\mid u^{\prime}(t)\right\| \|_{H}\right. \\
& +3 K_{a} T \max \|\mid u(t)\|\left\|_{H}+\left(2+3 K_{a} T\right)\right\|\left\|u_{0}\right\| \|_{H} \\
& \left.+3 T\|\| u_{1} \|\left.\right|_{H}\right)
\end{aligned}
$$

for each $t \in[0, T]$.

Remark In applications it is sometimes possible to use the Aubin-Nitche trick (see [15], [16]) to obtain higher order estimates for the projection error. But the improved projection errors serve no purpose if the interpolants are used to approximate the initial values $u_{0}$ and $u_{1}$. If the starting values $u_{0}^{h}$ and $u_{1}^{h}$ are projections of $u_{0}$ and $u_{1}$ with respect to the inner product $c$ (as in [1]), then the result above can be improved. This is in general not possible. 


\section{Fully discrete approximation}

\subsection{A system of ordinary differential equations}

In this section we consider the discretization of Problem $\mathrm{G}^{h}$. Suppose $S^{h}$ is the span of the set $\left\{\phi_{1}, \phi_{2}, \cdots, \phi_{n}\right\}$. Problem $\mathrm{G}^{h}$ is equivalent to a system of ordinary differential equations as we prove below.

\section{Notation}

Matrices $K, C, M$ and the vector $F$ are defined by

$$
K_{i j}=b\left(\phi_{j}, \phi_{i}\right), C_{i j}=a\left(\phi_{j}, \phi_{i}\right), M_{i j}=c\left(\phi_{j}, \phi_{i}\right) \text { and } F_{i}(t)=\left(f(t), \phi_{i}\right)_{X}
$$

If a function $w$ has values in $S^{h}$, i.e. $w=\sum_{j=1}^{n} w_{j} \phi_{j}$, then we define a function

$$
\bar{w}=\left(w_{1}, w_{2}, \ldots, w_{n}\right)=\left[\begin{array}{llll}
w_{1} & w_{2} & \ldots & w_{n}
\end{array}\right]^{t},
$$

with values in $R_{n}$. It is convenient to use $d^{h}$ and $v^{h}$ for initial conditions in this section instead of $u_{0}^{h}$ and $u_{1}^{h}$. We define the vectors $\bar{d}=\left(d_{1}, d_{2}, \ldots, d_{n}\right)$ and $\bar{v}=\left(v_{1}, v_{2}, \ldots, v_{n}\right)$ where $d^{h}=\sum_{j=1}^{n} d_{j} \phi_{j}$ and $v^{h}=\sum_{j=1}^{n} v_{j} \phi_{j}$.

\section{Problem ODE}

Determine $\bar{u} \in C^{2}(J)$ such that

$$
M \bar{u}^{\prime \prime}+C \bar{u}^{\prime}+K \bar{u}=F(t) \quad \text { with } \quad \bar{u}(0)=\bar{d} \text { and } \bar{u}^{\prime}(0)=\bar{v} .
$$

Proposition 6.1. Suppose $M, K, C, F, \bar{d}$ and $\bar{v}$ are defined as above. Then, the function $u_{h}$ is a solution of Problem $G^{h}$ if and only if the function $\bar{u}$ is a solution of Problem ODE.

Proposition 6.2. If $F \in C(J)$, then Problem ODE has a unique solution for each pair of vectors $\bar{d}$ and $\bar{v}$.

Proof. Since $c$ is an inner product, $M$ is invertible and the differential equation in Problem ODE may be written in the form $\bar{u}^{\prime \prime}+M^{-1} C \bar{u}^{\prime}+M^{-1} K \bar{u}=$ $M^{-1} F(t)$. It follows from the theory of linear differential equations that the initial value problem has a unique solution.

Proof of Theorem 3.1.

Clearly $F \in C(J)$ if $f \in C(J, X)$. The result follows from Propositions 6.1 and 6.2 . 
Remark For existence the continuity of $F$ is sufficient but for $\bar{u}$ to have derivatives of order $2+k$, we need to assume that $F$ has derivatives of order $k$. (Note that $F \in C^{k}(J)$ if $f \in C^{k}(J, X)$.)

\subsection{Fully discrete Galerkin scheme}

A finite difference method is used to approximate the solution of the system in Problem $\mathrm{G}^{h}$ (or Problem ODE). We follow [1] with an obvious modification to include the damping term. Suppose the interval $[0, T]$ is divided into $N$ steps of length $\tau=T / N$ and denote the approximation of $u_{h}\left(t_{k}\right)$ by $u_{k}^{h}$. Our aim is to estimate the difference between the solution of Problem $G$ and the fully discrete approximation

$$
u\left(t_{k}\right)-u_{k}^{h}=\left[u\left(t_{k}\right)-u_{h}\left(t_{k}\right)\right]+\left[u_{h}\left(t_{k}\right)-u_{k}^{h}\right] .
$$

An estimate for the error $u\left(t_{k}\right)-u_{h}\left(t_{k}\right)$ was obtained in Section 5 and an estimate for the error $u_{h}\left(t_{k}\right)-u_{k}^{h}$ is required. To be precise, an estimate for $\left\|u\left(t_{k}\right)-u_{h}\left(t_{k}\right)\right\|_{W}$ is derived in Theorem 5.2 and an estimate for the other error must be with respect to $\|\cdot\|_{W}$. Although norms are equivalent in a finite dimensional space, the dimension of $S^{h}$ is not fixed and equivalence of norms may not be used. For this reason we consider the fully discrete problem in variational form as in [3], [1] and [14].

Notation For any sequence $\left\{x_{k}\right\} \subset R_{n}$ :

$$
\begin{gathered}
\delta_{t} x_{k}=\tau^{-1}\left[x_{k+1}-x_{k}\right], \\
x_{k+\frac{1}{2}}=\frac{1}{2}\left[x_{k+1}+x_{k}\right] .
\end{gathered}
$$

\section{Problem $G^{h}-\mathrm{D}$}

Find a sequence $\left\{u_{k}^{h}\right\} \subset S^{h}$ such that for $k=0,1,2, \ldots, N-1$,

$$
\begin{aligned}
\delta_{t} u_{k}^{h} & =v_{k+\frac{1}{2}} \\
c\left(\delta_{t} v_{k}, \psi\right)+a\left(v_{k+\frac{1}{2}}, \psi\right)+b\left(u_{k+\frac{1}{2}}^{h}, \psi\right) & =\frac{1}{2}\left(\left[f\left(t_{k}\right)+f\left(t_{k+1}\right)\right], \psi\right)_{X}
\end{aligned}
$$

for each $\psi \in S^{h}$, while $u_{0}^{h}=u_{h}(0)=d^{h}$ and $v_{0}=u_{h}^{\prime}(0)=v^{h}$.

Following [1] we prove that the solution of Problem $G^{h}$-D is well defined. In the process an algorithm is derived. No confusion can arise if we write $u_{k}$ for $u_{k}^{h}$. 
Proposition 6.3. Problem $G^{h}$-D has a unique solution for any pair of vectors $d^{h}$ and $v^{h}$ in $S^{h}$.

Proof. For any $\psi \in S^{h}$,

$$
\begin{aligned}
\frac{\tau}{2} b\left(u_{k+1}+u_{k}, \psi\right) & =\frac{\tau^{2}}{2} b\left(\tau^{-1}\left[u_{k+1}-u_{k}\right], \psi\right)+\frac{\tau}{2} b\left(u_{k}, \psi\right)+\frac{\tau}{2} b\left(u_{k}, \psi\right) \\
& =\frac{\tau^{2}}{2} b\left(\frac{1}{2}\left[v_{k+1}+v_{k}\right], \psi\right)+\tau b\left(u_{k}, \psi\right) \\
& =\frac{\tau^{2}}{4} b\left(v_{k+1}, \psi\right)+\frac{\tau^{2}}{4} b\left(v_{k}, \psi\right)+\tau b\left(u_{k}, \psi\right) .
\end{aligned}
$$

Substitution into (6.2) yields

$$
\begin{aligned}
& c\left(v_{k+1}, \psi\right)-c\left(v_{k}, \psi\right)+\frac{\tau}{2} a\left(v_{k+1}+v_{k}, \psi\right)+\frac{\tau^{2}}{4} b\left(v_{k+1}, \psi\right)+\frac{\tau^{2}}{4} b\left(v_{k}, \psi\right)+\tau b\left(u_{k}, \psi\right) \\
& \quad=\frac{\tau}{2}\left(\left[f\left(t_{k}\right)+f\left(t_{k+1}\right)\right], \psi\right)_{X}
\end{aligned}
$$

or

$$
\begin{aligned}
c\left(v_{k+1}, \psi\right)+\frac{\tau}{2} a\left(v_{k+1}, \psi\right)+\frac{\tau^{2}}{4} b\left(v_{k+1}, \psi\right) \\
=c\left(v_{k}, \psi\right)-\frac{\tau}{2} a\left(v_{k}, \psi\right)-\frac{\tau^{2}}{4} b\left(v_{k}, \psi\right)-\tau b\left(u_{k}, \psi\right) \\
\quad+\frac{\tau}{2}\left(\left[f\left(t_{k}\right)+f\left(t_{k+1}\right)\right], \psi\right)_{X} .
\end{aligned}
$$

for any $\psi \in S^{h}$. The bilinear forms $b$ and $c$ are positive definite and as a consequence $c+\frac{\tau}{2} a+\frac{\tau^{2}}{4} b$ is positive definite. Therefore $v_{k+1}$ is uniquely determined by (6.3) and $u_{k+1}=u_{k}+\tau v_{k+\frac{1}{2}}$ from (6.1).

The following algorithm may be used.

\section{Problem FD}

Find a sequence $\left\{\bar{u}_{k}\right\} \subset R_{n}$ such that for each $k$,

$$
\begin{aligned}
\bar{u}_{k+1} & =\bar{u}_{k}+\tau \bar{v}_{k+\frac{1}{2}} \\
\left(M+\frac{\tau}{2} C+\frac{\tau^{2}}{4} K\right) \bar{v}_{k+1} & =\left(M-\frac{\tau}{2} C-\frac{\tau^{2}}{4} K\right) \bar{v}_{k}-\tau K \bar{u}_{k}+\frac{\tau}{2}\left[F\left(t_{k}\right)+F\left(t_{k+1}\right)\right]
\end{aligned}
$$

with $\bar{u}_{0}=\bar{d}$ and $\bar{v}_{0}=\bar{v}$. 


\subsection{Error estimates}

In this subsection we derive an estimate for the error $u_{h}\left(t_{k}\right)-u_{k}^{h}$. Recall that it must be with respect to $\|\cdot\|_{W}$. Usually the error $u\left(t_{k}\right)-u_{k}^{h}$ is estimated directly, see e.g. [3], [1], [14], [5], [6] and [7]. To do this, the assumption is made that the exact solution has derivatives $u^{(k)} \in \mathcal{L}^{2}([0, T], V)$ for $k \leq 4$ or $k \leq 3$. This assumption is very restrictive.

To obtain our general result, we adapt the proof in [1] (which is for the wave equation).

\section{Truncation}

Consider Problem $G^{h}$-D. Substituting $t=t_{k}$ and $t=t_{k+1}$ respectively into Problem $\mathrm{G}^{h}$ (Equation (3.1)), we obtain

$$
\begin{gathered}
c\left(\tau^{-1}\left[v_{h}\left(t_{k+1}\right)-v_{h}\left(t_{k}\right)\right], \psi\right)+\frac{1}{2} a\left(\left[v_{h}\left(t_{k+1}\right)+v_{h}\left(t_{k}\right)\right], \psi\right)+\frac{1}{2} b\left(\left[u_{h}\left(t_{k+1}\right)+u_{h}\left(t_{k}\right)\right], \psi\right) \\
=\frac{1}{2}\left(\left[f\left(t_{k+1}\right)+f\left(t_{k}\right)\right], \psi\right)_{X}+c\left(\rho_{k}, \psi\right)
\end{gathered}
$$

where $v_{h}(t)=u_{h}^{\prime}(t)$ and

$$
\rho_{k}=\tau^{-1}\left[v_{h}\left(t_{k+1}\right)-v_{h}\left(t_{k}\right)\right]-\frac{1}{2}\left[v_{h}^{\prime}\left(t_{k+1}\right)+v_{h}^{\prime}\left(t_{k}\right)\right] .
$$

We denote the errors by $e_{k}$ and $q_{k}$ :

$$
e_{k}=u_{h}\left(t_{k}\right)-u_{k} \quad \text { and } \quad q_{k}=u_{h}^{\prime}\left(t_{k}\right)-v_{k} .
$$

Note that in our approach $e_{0}=0$ and $q_{0}=0$.

By subtracting (6.2) from (6.4) we obtain

$$
c\left(\tau^{-1}\left[q_{k+1}-q_{k}\right], \psi\right)+a\left(\frac{1}{2}\left[q_{k+1}+q_{k}\right], \psi\right)+b\left(\frac{1}{2}\left[e_{k+1}+e_{k}\right], \psi\right)=c\left(\rho_{k}, \psi\right),
$$

which can be rewritten as follows

$$
c\left(\delta_{t} q_{k}, \psi\right)+a\left(q_{k+\frac{1}{2}}, \psi\right)+b\left(e_{k+\frac{1}{2}}, \psi\right)=c\left(\rho_{k}, \psi\right) .
$$

Substitution of $u_{h}$ and $v_{h}$ into (6.1) yields

$$
\tau^{-1}\left[u_{h}\left(t_{k+1}\right)-u_{h}\left(t_{k}\right)\right]=\frac{1}{2}\left(\left[v_{h}\left(t_{k+1}\right)+v_{h}\left(t_{k}\right)\right]+\sigma_{k},\right.
$$


where (obviously)

$$
\sigma_{k}=\tau^{-1}\left[u_{h}\left(t_{k+1}\right)-u_{h}\left(t_{k}\right)\right]-\frac{1}{2}\left(\left[v_{h}\left(t_{k+1}\right)+v_{h}\left(t_{k}\right)\right] .\right.
$$

It follows from (6.1) and (6.7) that

$$
\delta_{t} e_{k}=q_{k+\frac{1}{2}}+\sigma_{k}
$$

\section{Stability}

Consider any integer $n>0$. It follows from (6.9) that

$$
e_{n}=\tau \sum_{k=0}^{n-1} \delta_{t} e_{k}=\tau \sum_{k=0}^{n-1} q_{k+\frac{1}{2}}+\tau \sum_{k=0}^{n-1} \sigma_{k} .
$$

Consequently

$$
\begin{aligned}
e_{n+\frac{1}{2}} & =\frac{1}{2}\left(e_{n+1}+e_{n}\right) \\
& =\frac{\tau}{2}\left(\sum_{k=0}^{n} q_{k+\frac{1}{2}}+\sum_{k=0}^{n-1} q_{k+\frac{1}{2}}\right)+\frac{\tau}{2}\left(\sum_{k=0}^{n} \sigma_{k}+\sum_{k=0}^{n-1} \sigma_{k}\right) .
\end{aligned}
$$

But from (6.9) we also have

$$
\begin{aligned}
\delta_{t} e_{n} & =\frac{1}{2}\left[q_{n+1}+q_{n}\right]+\sigma_{n} \\
& =\frac{\tau}{2}\left[\sum_{k=0}^{n} \delta_{t} q_{k}+\sum_{k=0}^{n-1} \delta_{t} q_{k}\right]+\sigma_{n} .
\end{aligned}
$$

Let $s_{0}=0$ and $s_{n}=\tau \sum_{k=0}^{n-1} e_{k+\frac{1}{2}}$, then

$$
s_{n+\frac{1}{2}}=\frac{\tau}{2}\left[\sum_{k=0}^{n} e_{k+\frac{1}{2}}+\sum_{k=0}^{n-1} e_{k+\frac{1}{2}}\right] \text {. }
$$

In the analysis that follows, $\psi$ denotes an arbitrary element of $S^{h}$. Using 
(6.10), (6.11) and (6.12), we have for $n=1,2, \ldots, N-1$

$$
\begin{aligned}
& c\left(\delta_{t} e_{n}, \psi\right)+a\left(e_{n+\frac{1}{2}}, \psi\right)+b\left(s_{n+\frac{1}{2}}, \psi\right)=\frac{\tau}{2} c\left(\left[\sum_{k=0}^{n} \delta_{t} q_{k}+\sum_{k=0}^{n-1} \delta_{t} q_{k}\right], \psi\right)+c\left(\sigma_{n}, \psi\right) \\
& \quad+\frac{\tau}{2} a\left(\left[\sum_{k=0}^{n} q_{k+\frac{1}{2}}+\sum_{k=0}^{n-1} q_{k+\frac{1}{2}}\right], \psi\right) \\
& \quad+\frac{\tau}{2} b\left(\left[\sum_{k=0}^{n} e_{k+\frac{1}{2}}+\sum_{k=0}^{n-1} e_{k+\frac{1}{2}}\right], \psi\right) .
\end{aligned}
$$

Using (6.6), it follows that

$$
\begin{aligned}
& c\left(\delta_{t} e_{n}, \psi\right)+a\left(e_{n+\frac{1}{2}}, \psi\right)+b\left(s_{n+\frac{1}{2}}, \psi\right) \\
& =\frac{\tau}{2} c\left(\left[\sum_{k=0}^{n} \rho_{k}+\sum_{k=0}^{n-1} \rho_{k}\right], \psi\right)+c\left(\sigma_{n}, \psi\right) .
\end{aligned}
$$

Let

$$
\epsilon_{n}=\frac{\tau}{2} \rho_{n}+\tau \sum_{k=0}^{n-1} \rho_{k}+\sigma_{n}
$$

for $n=1,2, \ldots N-1$, then

$$
c\left(\delta_{t} e_{n}, \psi\right)+a\left(e_{n+\frac{1}{2}}, \psi\right)+b\left(s_{n+\frac{1}{2}}, \psi\right)=c\left(\epsilon_{n}, \psi\right) .
$$

Choose $\psi=e_{n+\frac{1}{2}}$, then

$$
\begin{array}{r}
\left\|e_{n+1}\right\|^{2}-\left\|e_{n}\right\|^{2}+2 \tau a\left(e_{n+\frac{1}{2}}, e_{n+\frac{1}{2}}\right)+\left\|s_{n+1}\right\|_{V}^{2}-\left\|s_{n}\right\|_{V}^{2} \\
=2 \tau c\left(\epsilon_{n}, e_{n+\frac{1}{2}}\right) .
\end{array}
$$

Consider any integer $\nu$ with $2 \leq \nu \leq N$. Using (6.15) and the fact that $a\left(e_{n+\frac{1}{2}}, e_{n+\frac{1}{2}}\right) \geq 0$, we have

$$
\sum_{n=1}^{\nu-1}\left[\left\|e_{n+1}\right\|^{2}-\left\|e_{n}\right\|^{2}+\left\|s_{n+1}\right\|_{V}^{2}-\left\|s_{n}\right\|_{V}^{2}\right] \leq 2 \tau \sum_{n=1}^{\nu-1} c\left(\epsilon_{n}, e_{n+\frac{1}{2}}\right) .
$$


It follows that

$$
\begin{aligned}
& \left\|e_{\nu}\right\|_{W}^{2}-\left\|e_{1}\right\|_{W}^{2}+\left\|s_{\nu}\right\|_{V}^{2}-\left\|s_{1}\right\|_{V}^{2} \\
& \quad \leq 4 T \tau \sum_{n=1}^{\nu-1}\left\|\epsilon_{n}\right\|_{W}^{2}+\frac{\tau}{4 T} \sum_{n=1}^{\nu-1}\left\|e_{n+\frac{1}{2}}\right\|_{W}^{2}
\end{aligned}
$$

using the Cauchy-Schwartz inequality and Young's inequality.

Now, consider $n=0$. Let $k=0$ in (6.6):

$$
c\left(\delta_{t} q_{0}, \psi\right)+a\left(q_{\frac{1}{2}}, \psi\right)+b\left(e_{\frac{1}{2}}, \psi\right)=c\left(\rho_{0}, \psi\right) .
$$

Also, let $k=0$ in (6.9):

$$
\delta_{t} e_{0}=q_{\frac{1}{2}}+\sigma_{0}
$$

Since $q_{0}=0$ and $e_{0}=0$ we have $\delta_{t} e_{0}=\frac{e_{1}}{\tau}, e_{\frac{1}{2}}=\frac{e_{1}}{2}, \delta_{t} q_{0}=\frac{q_{1}}{\tau}$ and $q_{\frac{1}{2}}=\frac{q_{1}}{2}$ and hence

$$
q_{1}=\frac{2}{\tau} e_{1}-2 \sigma_{0}
$$

Making the appropriate substitutions into (6.17) we obtain

$$
\begin{aligned}
& \frac{2}{\tau^{2}} c\left(e_{1}, \psi\right)+\frac{1}{\tau} a\left(e_{1}, \psi\right)+\frac{1}{2} b\left(e_{1}, \psi\right) \\
& =c\left(\rho_{0}, \psi\right)+\frac{2}{\tau} c\left(\sigma_{0}, \psi\right)+a\left(\sigma_{0}, \psi\right) .
\end{aligned}
$$

From the definition of $s_{0}$ and $s_{1}$, we have $e_{1}=\frac{2}{\tau} s_{1}$. Substituting this into the bilinear form $b$ in (6.18) (and multiplying by $\frac{\tau^{2}}{2}$ ) yields

$$
c\left(e_{1}, \psi\right)+\frac{\tau}{2} a\left(e_{1}, \psi\right)+\frac{\tau}{2} b\left(s_{1}, \psi\right)=\frac{\tau^{2}}{2} c\left(\rho_{0}, \psi\right)+\tau c\left(\sigma_{0}, \psi\right)+\frac{\tau^{2}}{2} a\left(\sigma_{0}, \psi\right) .
$$

Choose $\psi=e_{1}$, then

$$
\begin{aligned}
& \left\|e_{1}\right\|_{W}^{2}+\left\|s_{1}\right\|_{V}^{2}+\frac{\tau}{2} a\left(e_{1}, e_{1}\right) \\
& \quad=\frac{\tau^{2}}{2} c\left(\rho_{0}, e_{1}\right)+\tau c\left(\sigma_{0}, e_{1}\right)+\frac{\tau^{2}}{2} a\left(\sigma_{0}, e_{1}\right) .
\end{aligned}
$$


Since $a\left(e_{1}, e_{1}\right) \geq 0$,

$$
\begin{aligned}
\left\|e_{1}\right\|_{W}^{2}+\left\|s_{1}\right\|_{V}^{2} \leq & \frac{\tau^{2}}{2}\left\|\rho_{0}\right\|_{W}\left\|e_{1}\right\|_{W}+\tau\left\|\sigma_{0}\right\|_{W}\left\|e_{1}\right\|_{W}+\frac{\tau^{2}}{2} K_{a}\left\|\sigma_{0}\right\|_{W}\left\|e_{1}\right\|_{W} \\
\leq & \tau^{4}\left\|\rho_{0}\right\|_{W}^{2}+\frac{1}{16}\left\|e_{1}\right\|_{W}^{2}+4 \tau^{2}\left\|\sigma_{0}\right\|_{W}^{2}+\frac{1}{16}\left\|e_{1}\right\|_{W}^{2} \\
& \quad+\tau^{4} K_{a}^{2}\left\|\sigma_{0}\right\|_{W}^{2}+\frac{1}{16}\left\|e_{1}\right\|_{W}^{2} \\
= & \frac{3}{16}\left\|e_{1}\right\|_{W}^{2}+\tau^{4}\left\|\rho_{0}\right\|_{W}^{2}+\left(4 \tau^{2}+\tau^{4} K_{a}\right)\left\|\sigma_{0}\right\|_{W}^{2}
\end{aligned}
$$

using the Cauchy-Schwartz and Young's inequalities.

To proceed, we combine (6.16) and (6.19):

$$
\begin{aligned}
\left\|e_{\nu}\right\|_{W}^{2} \leq 4 T \tau & \sum_{n=1}^{\nu-1}\left\|\epsilon_{n}\right\|_{W}^{2}+\frac{\tau}{4 T} \sum_{n=1}^{\nu-1}\left\|e_{n+\frac{1}{2}}\right\|_{W}^{2} \\
& +\frac{3}{16}\left\|e_{1}\right\|_{W}^{2}+\tau^{4}\left\|\rho_{0}\right\|_{W}^{2}+\left(4 \tau^{2}+\tau^{4} K_{a}\right)\left\|\sigma_{0}\right\|_{W}^{2} \\
\leq & 4 T \tau \sum_{n=1}^{\nu-1}\left\|\epsilon_{n}\right\|_{W}^{2}+\frac{1}{2} \max \left\|e_{n}\right\|_{W}^{2}+\tau^{4}\left\|\rho_{0}\right\|_{W}^{2}+\left(4 \tau^{2}+\tau^{4} K_{a}\right)\left\|\sigma_{0}\right\|_{W}^{2} .
\end{aligned}
$$

The stability result follows.

\section{Lemma 6.1. Stability}

$$
\max \left\|e_{n}\right\|_{W}^{2} \leq 8 T \tau \sum_{n=0}^{N-1}\left\|\epsilon_{n}\right\|_{W}^{2}+2 \tau^{4}\left\|\rho_{0}\right\|_{W}^{2}+\left(8 \tau^{2}+2 \tau^{4} K_{a}\right)\left\|\sigma_{0}\right\|_{W}^{2} .
$$

\section{Convergence}

It remains to determine estimates for $\rho_{k}$ and $\sigma_{k}$. To find an estimate for $\rho_{k}$, we consider the following two parts separately

$$
\begin{aligned}
\rho_{A} & =\tau^{-1}\left[v_{h}\left(t_{k+1}\right)-v_{h}\left(t_{k}\right)\right]-v_{h}^{\prime}\left(t_{k}+\frac{\tau}{2}\right) \text { and } \\
\rho_{B} & =-\frac{1}{2}\left[v_{h}^{\prime}\left(t_{k+1}\right)+v_{h}^{\prime}\left(t_{k}\right)\right]+v_{h}^{\prime}\left(t_{k}+\frac{\tau}{2}\right) .
\end{aligned}
$$

Recall that $v_{h}\left(t_{k}\right)=\sum_{i=1}^{n} v_{i}\left(t_{k}\right) \phi_{i}$. Using Taylor expansions we obtain

$$
\begin{aligned}
\tau^{-1} & {\left[v_{i}\left(t_{k+1}\right)-v_{i}\left(t_{k}\right)\right]-v_{i}^{\prime}\left(t_{k}+\frac{\tau}{2}\right) } \\
& =\frac{1}{4 \tau} \int_{t_{k}+\frac{\tau}{2}}^{t_{k+1}}\left(t_{k+1}-\theta\right)^{2} v_{i}^{\prime \prime \prime}(\theta) d \theta+\frac{1}{4 \tau} \int_{t_{k}}^{t_{k}+\frac{\tau}{2}}\left(t_{k}-\theta\right)^{2} v_{i}^{\prime \prime \prime}(\theta) d \theta .
\end{aligned}
$$


From the Cauchy-Schwartz inequality

$$
\begin{aligned}
\left(\int_{t_{k}+\frac{\tau}{2}}^{t_{k+1}}\left(t_{k+1}-\theta\right)^{2} v_{i}^{\prime \prime \prime}(\theta) d \theta\right)^{2} & \leq \int_{t_{k}+\frac{\tau}{2}}^{t_{k+1}}\left(t_{k+1}-\theta\right)^{4} d \theta \int_{t_{k}+\frac{\tau}{2}}^{t_{k+1}}\left(v_{i}^{\prime \prime \prime}(\theta)\right)^{2} d \theta \\
& =\frac{\tau^{5}}{160} \int_{t_{k}+\frac{\tau}{2}}^{t_{k+1}}\left(v_{i}^{\prime \prime \prime}(\theta)\right)^{2} d \theta \quad \text { and } \\
\left(\int_{t_{k}}^{t_{k}+\frac{\tau}{2}}\left(t_{k}-\theta\right)^{2} v_{i}^{\prime \prime \prime}(\theta) d \theta\right)^{2} & \leq \frac{\tau^{5}}{160} \int_{t_{k}}^{t_{k}+\frac{\tau}{2}}\left(v_{i}^{\prime \prime \prime}(\theta)\right)^{2} d \theta
\end{aligned}
$$

Using the estimate above and assuming that $\left\{\phi_{j}\right\}$ is orthonormal, we have

$$
\begin{aligned}
\left\|\rho_{A}\right\|_{W}^{2} & =\left\|\tau^{-1}\left[v_{h}\left(t_{k+1}\right)-v_{h}\left(t_{k}\right)\right]-v_{h}^{\prime}\left(t_{k}+\frac{\tau}{2}\right)\right\|_{W}^{2} \\
& \leq \sum_{i=1}^{n} 2\left(\frac{1}{4 \tau} \int_{t_{k}+\frac{\tau}{2}}^{t_{k+1}}\left(t_{k+1}-\theta\right)^{2} v_{i}^{\prime \prime \prime}(\theta) d \theta\right)^{2}+\sum_{i=1}^{n} 2\left(\frac{1}{4 \tau} \int_{t_{k}}^{t_{k}+\frac{\tau}{2}}\left(t_{k}-\theta\right)^{2} v_{i}^{\prime \prime \prime}(\theta) d \theta\right)^{2} \\
& \left.\leq \frac{\tau^{3}}{1280}\left[\int_{t_{k}+\frac{\tau}{2}}^{t_{k+1}}\left\|v_{h}^{\prime \prime \prime}(\theta)\right\|_{W}^{2} d \theta+\int_{t_{k}}^{t_{k}+\frac{\tau}{2}} \| v_{h}^{\prime \prime \prime}(\theta)\right) \|_{W}^{2} d \theta\right] \\
& \leq \frac{\tau^{4}}{1280} \max \left\|v_{h}^{\prime \prime \prime}\right\|_{W}^{2},
\end{aligned}
$$

where $\max _{\theta \in[0, T]}$ is denoted by max.

To find an estimate for $\rho_{B}$, the same steps are followed.

From Taylor expansions

$$
\begin{aligned}
& \frac{1}{2}\left[v_{i}^{\prime}\left(t_{k+1}\right)+v_{i}^{\prime}\left(t_{k}\right)\right]-v_{i}^{\prime}\left(t_{k}+\frac{\tau}{2}\right) \\
& \quad=\frac{1}{2} \int_{t_{k}+\frac{\tau}{2}}^{t_{k+1}}\left(t_{k+1}-\theta\right) v_{i}^{\prime \prime \prime}(\theta) d \theta-\frac{1}{2} \int_{t_{k}}^{t_{k}+\frac{\tau}{2}}\left(t_{k}-\theta\right) v_{i}^{\prime \prime \prime}(\theta) d \theta .
\end{aligned}
$$

Using the Cauchy-Schwartz inequality again, we obtain

$$
\begin{aligned}
\left(\int_{t_{k}+\frac{\tau}{2}}^{t_{k+1}}\left(t_{k+1}-\theta\right) v_{i}^{\prime \prime \prime}(\theta) d \theta\right)^{2} & \leq \int_{t_{k}+\frac{\tau}{2}}^{t_{k+1}}\left(t_{k+1}-\theta\right)^{2} d \theta \int_{t_{k}+\frac{\tau}{2}}^{t_{k+1}}\left(v_{i}^{\prime \prime \prime}(\theta)\right)^{2} d \theta \\
& =\frac{\tau^{3}}{24} \int_{t_{k}+\frac{\tau}{2}}^{t_{k+1}}\left(v_{i}^{\prime \prime \prime}(\theta)\right)^{2} d \theta \quad \text { and } \\
\left(\int_{t_{k}}^{t_{k}+\frac{\tau}{2}}\left(t_{k}-\theta\right)^{2} v_{i}^{(4)}(\theta) d \theta\right)^{2} & \leq \frac{\tau^{3}}{24} \int_{t_{k}}^{t_{k}+\frac{\tau}{2}}\left(v_{i}^{\prime \prime \prime}(\theta)\right)^{2} d \theta
\end{aligned}
$$


Therefore

$$
\left\|\rho_{B}\right\|_{W}^{2} \leq \frac{\tau^{4}}{48} \max \left\|v_{h}^{\prime \prime \prime}\right\|_{W}^{2}
$$

Combining the estimates for $\rho_{A}$ and $\rho_{B}$ yields

$$
\left\|\rho_{k}\right\|_{W}^{2} \leq \tau^{4} \max \left\|v_{h}^{\prime \prime \prime}\right\|_{W}^{2} .
$$

Now consider $\sigma_{k}=\tau^{-1}\left[u_{h}\left(t_{k+1}\right)-u_{h}\left(t_{k}\right)\right]-\frac{1}{2}\left[u_{h}^{\prime}\left(t_{k+1}\right)+u_{h}^{\prime}\left(t_{k}\right)\right]$. Following the same method as for $\rho_{k}$, we obtain

$$
\left\|\sigma_{k}\right\|_{W}^{2} \leq \tau^{4} \max \left\|u_{h}^{\prime \prime \prime}\right\|_{W}^{2} .
$$

The following estimate for $\epsilon_{n}$ (see (6.14)) is obtained from (6.20) and (6.21).

$$
\left\|\epsilon_{n}\right\|_{W}^{2} \leq 5 T^{2} \tau^{4} \max \left\|v_{h}^{\prime \prime \prime}\right\|_{W}^{2}+4 \tau^{4} \max \left\|u_{h}^{\prime \prime \prime}\right\|_{W}^{2} .
$$

Using the result in Lemma 6.1 yields

$$
\begin{aligned}
\max \left\|e_{n}\right\|_{W}^{2} \leq \quad & 40 T^{4} \tau^{4} \max \left\|v_{h}^{\prime \prime \prime}\right\|_{W}^{2}+32 T^{2} \tau^{4} \max \left\|u_{h}^{\prime \prime \prime}\right\|_{W}^{2} \\
& +2 \tau^{8} \max \left\|v_{h}^{\prime \prime \prime}\right\|_{W}^{2}+8 \tau^{6} \max \left\|u_{h}^{\prime \prime \prime}\right\|_{W}^{2}+2 \tau^{8} K_{a} \max \left\|u_{h}^{\prime \prime \prime}\right\|_{W}^{2} .
\end{aligned}
$$

Note that if $f \in C^{2}([0, T], X)$, then $u_{h} \in C^{4}([0, T], W)$ and consequently the following error estimate is obtained.

Theorem 6.1. Error estimate.

If $f \in C^{2}([0, T], X)$, then

$$
\begin{gathered}
\left\|u_{h}\left(t_{k}\right)-u_{k}^{h}\right\|_{W} \leq 7 T^{2} \tau^{2} \max \left\|u_{h}^{(4)}\right\|_{W}+7 T \tau^{2} \max \left\|u_{h}^{\prime \prime \prime}\right\|_{W} \\
+\sqrt{2 K_{a}} \tau^{4} \max \left\|u_{h}^{\prime \prime \prime}\right\|_{W}
\end{gathered}
$$

for each $t \in(0, T)$.

Finally, error estimates for the fully discrete approximation of the solution of Problem G are obtained by combining results from Section 5 with Theorem 6.1. 


\section{Applications}

\section{The multi-dimensional wave equation}

As an elementary application we consider the wave equation in a two-dimensional bounded domain denoted by $\Omega$. Let $\Sigma$ be a part of the boundary $\partial \Omega$. Given functions $f, u_{0}$ and $u_{1}$, find $u$ defined on $\bar{\Omega} \times[0, T]$ such that

$$
\begin{aligned}
\rho \partial_{t}^{2} u & =\nabla \cdot(A \nabla u)-k \partial_{t} u+f \quad \text { in } \Omega \times(0, T), \\
u & =0 \quad \text { on } \partial \Omega-\Sigma, \\
-A \nabla u \cdot n & =0 \text { on } \Sigma,
\end{aligned}
$$

while $u(\cdot, 0)=u_{0}$ and $\partial_{t} u(\cdot, 0)=u_{1}$.

The unit outer normal on the boundary is denoted by $n$. The given parameters in the problem are the matrix of functions $A=\left(a_{i j}\right)$ and the functions $k$ and $\rho$. We assume that the parameters are bounded above and below by positive numbers and the matrix $\mathrm{A}$ is uniformly positive definite. For the problem in [1], $\Sigma$ is empty and $k=0$.

Next consider the variational form. The test functions $T(\Omega)$ are functions in $C^{1}(\bar{\Omega})$ which vanish on $\partial \Omega-\Sigma$. Let $(f, g)$ denote the usual innerproduct for $\mathcal{L}^{2}(\Omega)$. The bilinear forms $a, b$ and $c$ are defined as follows (using weak derivatives for $b$ )

$$
\begin{aligned}
b(u, v) & =\iint_{\Omega} A \nabla u \cdot \nabla v d V \\
c(u, v) & =\iint_{\Omega} \rho u v d V \\
a(u, v) & =\iint_{\Omega} k u v d V .
\end{aligned}
$$

Let $V$ denote the closure of $T(\Omega)$ in $H^{1}(\Omega)$. The weak variational form of the problem is to find $u$ such that for each $t>0, u(t) \in V, u^{\prime \prime}(t) \in \mathcal{L}^{2}(\Omega)$ and

$$
c\left(u^{\prime \prime}(t), v\right)+a\left(u^{\prime}(t), v\right)+b(u(t), v)=(f(t), v), \text { for each } v \in V
$$

while $u(0)=u_{0}$ and $u^{\prime}(0)=u_{1}$.

Note that the restriction to two dimensions is not necessary. Next we show that Assumptions E1, E2, E3 and E4 are satisfied. 
E1: $V$ is dense in $\mathcal{L}^{2}(\Omega)$ since $C_{0}^{\infty}(\Omega)$ is dense in $\mathcal{L}^{2}(\Omega)$.

E2: Assuming that $\partial \Omega-\Sigma$ has positive length, the Poincaré-Friedrichs inequality can be proved and it follows that $b$ is positive definite. The norm corresponding to the inner product $b$ is $\|v\|_{V}=\sqrt{b(v, v)}$. It is equivalent to the norm of $H^{1}(\Omega)$ on $V$.

E3: The bilinear form $c$ is clearly an inner product for $X=\mathcal{L}^{2}(\Omega)$ and the corresponding norm is equivalent to the norm of $\mathcal{L}^{2}(\Omega)$. The space $\mathcal{L}^{2}(\Omega)$ with inner product $c$ is the space $\mathrm{W}$ (with norm $\|\cdot\|_{W}$ ).

E4: Clearly $a$ is defined on $\mathcal{L}^{2}(\Omega)$ and is bounded.

Existence of a unique solution for the weak variational problem follows from Theorem 2.1, provided that $f \in C^{1}\left([0, T], \mathcal{L}^{2}(\Omega)\right)$.

Now consider the finite element approximation of the problem. For simplicity we consider the two-dimensional case and use triangle elements. A finite dimensional subspace $S^{h}$ of $V$ is constructed, using piecewise linear basis functions.

The Galerkin approximation is to find a function $u_{h} \in C^{2}(0, T) \cap C^{1}[0, T]$ such that

$$
c\left(u_{h}^{\prime \prime}(t), v\right)+a\left(u_{h}^{\prime}(t), v\right)+b\left(u_{h}(t), v\right)=(f(\cdot, t), v)_{X},
$$

for each $v \in S^{h}$, while $u_{h}(0)=u_{0}^{h}$ and $u_{h}^{\prime}(0)=u_{1}^{h}$.

Depending on the properties of the boundary of $\Omega$, the parameters, initial values $u_{0}$ and $u_{1}$ and the function $f$, it can be proved that A1 and A2 hold. (It is beyond the scope of this article.) For the problem under discussion we have that $H=H^{k}(\Omega) \cap V$ (see Section 5). Instead of Assumption A2 we have the following result for piecewise linear basis functions: If $u \in H^{k}(\Omega)$ for $k \geq 2$, then

$$
\|\Pi u-u\|_{V} \leq \widehat{C}_{T} h|u|_{2}
$$

where $|\cdot|_{2}$ denotes the usual semi-norm.

Consider application of Theorem 5.2. For the present problem we have

$$
\|u-P u\|_{W} \leq C_{b}\|u-P u\|_{V} \leq \widehat{C}_{b} C_{T} h|u|_{2} .
$$

\section{Error estimate 7.1}

Suppose $u_{0}^{h}=\Pi u_{0}$ and $u_{1}^{h}=\Pi u_{1}$. If the solution $u$ of Problem G satisfies 
Assumption A1, $u(t) \in H^{2}(\Omega)$ and $u^{\prime}(t) \in H^{2}(\Omega)$, then

$$
\begin{aligned}
\left\|u(t)-u_{h}(t)\right\|_{W} \leq & C_{b} \widehat{C}_{T} h|u(t)|_{2}+\sqrt{2} C_{b} \widehat{C}_{T} h\left(3 T \max \left|u^{\prime}(t)\right|_{2}\right. \\
& \left.+3 K_{a} T \max |u(t)|_{2}+\left(2+3 K_{a} T\right)\left|u_{0}\right|_{2}+3 T\left|u_{1}\right|_{2}\right),
\end{aligned}
$$

for each $t \in[0, T]$.

If the solution is sufficiently smooth, i.e. $E_{b} \subset H^{2}(\Omega)$, the Aubin-Nitche trick may be used. Instead of $(7.1)$ we have $\|u-P u\|_{W} \leq \widehat{K} h^{2}|u|_{2}$ where $\widehat{K}$ depends on $C_{b}$ and $\widehat{C}_{T}$. Using this estimate in Theorem 5.1, we obtain the following result.

\section{Error estimate 7.2}

Let $u_{0}^{h}=P u_{0}$ and $u_{1}^{h}=P u_{1}$. Assume that $u(t)$ and $u^{\prime}(t)$ are in $H^{2}(\Omega)$. Then,

$$
\begin{aligned}
\left\|u(t)-u_{h}(t)\right\|_{W} \leq & \widehat{K} h^{2}|u(t)|_{2}+\sqrt{2} \widehat{K} h^{2}\left(3 T \max \left|u^{\prime}(t)\right|_{2}\right. \\
& \left.+3 K_{a} T \max |u(t)|_{2}+\left(2+3 K_{a} T\right)\left|u_{0}\right|_{2}+3 T\left|u_{1}\right|_{2}\right),
\end{aligned}
$$

for each $t \in[0, T]$.

From the definition of the spaces $X, W$ and $V$ and the bilinear forms $c, a$ and $b$, it follows that the theory of Section 6 is applicable. Combining either Estimate 7.1 or 7.2 with Theorem 6.1 yields the error estimate for the fully discrete approximation.

\section{Elasto-dynamics, hybrid models and structures}

The theory in this article can also be applied to the Timoshenko beam model (with viscous type damping) [5], the Reissner-Mindlin plate model (undamped) [17] and linear elasto-dynamics (undamped) [7].

The theory of this article is really intended for systems where elastic bodies interact. Numerous examples can be found in [18], for example networks of linked beams.

Another example where the theory may be applied, is in article [19] where the importance of vibration analysis of structures in engineering is stressed. The authors consider a system consisting of two plates.

A hybrid model where a beam has complex boundary conditions due to a damping tip body attached to one end is considered in [20]. The damping is not of viscous type and the theory in this article does not apply, but it does for the undamped hybrid model. 
Perhaps the most convenient example for the present discussion, is in a platebeam model from [21]. The model problem referred to as Problem RMT, is of the same type as Problem G. If the damping in a joint is replaced by viscous damping on the plate, then Problem RMT is a special case of Problem G. It is proved in [21] that assumptions E1, E2 and E3 are satisfied. For viscous damping on the plate, Assumption E4 is easy to verify.

Finally, we mention the rather obvious fact that other types of damping need to be considered. Considering the existence results for different types of damping in [11], we believe that mere adjustments to the present article will not yield the desired results.

Acknowledgement This research was supported in part by NRF (South Africa) grant IFR2011041700007.

\section{References}

[1] G.A. Baker (1976). Error estimates for finite element methods for second order hyperbolic equations. SIAM J. Numer. Anal. 13:564576.

[2] G. Strang and G.J. Fix (1973). An Analysis of the Finite Element Method. Prentice-Hall, New Jersey.

[3] T. Dupont (1973). $\mathcal{L}^{2}$-estimates for Galerkin methods for second order hyperbolic equations. SIAM J. Numer. Anal. 10:880-889.

[4] L.C. Cowsar, T.F. Dupont and M.F. Wheeler (1990). A priory estimates for mixed finite element methods for the wave equation. Comput. Methods Appl. Mech. Engrg. 82:205-222.

[5] B. Semper (1994). Semi-discrete and fully discrete Galerkin methods for the vibrating Timoshenko beam. Comput. Methods Appl. Mech. Engrg. 117:353-360.

[6] M. Feng, X. Xie and H. Xiong (1999). Semi-discrete and fully discrete partial projection finite element methods for the vibrating Timoshenko beam. J. Comput. Math. 17:353-368. 
[7] S.R. Wu (2003). A priori error estimates for explicit finite element for linear elasto-dynamics by Galerkin method for central difference method. Comput. Methods Appl. Mech. Engrg. 192:5329-5353.

[8] M.J. Grote, A. Schneebeli and D. Schötzau (2006). Discontinuous Galerkin finite element method for the wave equation. SIAM J. $\mathrm{Nu}$ mer. Anal. 44:2408-2431.

[9] L. Zietsman, A.J. Van Der Merwe and N.F.J. Van Rensburg (1999). Convergence of the FEM solution for the vibration of a damaged beam. Technical report UPWT 99/20. Pretoria: Department of Mathematics and Applied Mathematics, University of Pretoria.

[10] L. Zietsman (2000). Finite element analysis of vibration models with interface conditions, $\mathrm{PhD}$ thesis, University of Pretoria.

[11] N.F.J. Van Rensburg and A.J. Van Der Merwe (2002). Analysis of the solvability of linear vibration models. Appl. Anal. 81:1143-1159.

[12] J.L Lions and E. Magenes (1972). Nonhomogeneous Bounday Value Problems and Applications, vol.1. Springer, New York.

[13] R.E. Showalter (1977). Hilbert space methods for partial differential equations. Pitman, London.

[14] J.T. Oden and J.N Reddy (1976). An introduction to the mathematical theory of finite elements. John Wiley and Sons, New York-LondonSydney-Toronto.

[15] J.P. Aubin (1967). Behaviour of the error for the approximate solution of boundary value problems for linear elliptic operators by Galerkin's and finite difference methods. Annali della Scuola Normale di Pisa, Series 3. 21:599-637.

[16] J. Nitsche (1969). Ein Kriterium fur die Quasi-Optimalitat dis ritzchen Verfahrens. Numer. Math. 11:346-348.

[17] S.R. Wu (2005). A priori error estimation of a four-node ReissnerMindlin plate element for elasto-dynamics. Comput. Methods Appl. Mech. Engrg. 194:2257-2281.

[18] J.E. Lagnese, G. Leugering and E.J.P.G. Schmidt (1994). Modeling Analysis and Control of Dynamic Elastic Multi-Link Structures. Birkhäuser, Boston. 
[19] J. Lai and J. Huang (2009). A finite element method for vibration analysis of elastic plate-plate structures. Discrete Contin. Dyn. Syst. Ser. B 11:387-419.

[20] K.T. Andrews and Shillor (2002). Vibrations of a beam with a damping tip body. Math. Comput. Modeling. 35:1033-1042.

[21] N.F.J. Van Rensburg, L. Zietsman and A.J. Van Der Merwe (2009). Solvability of a Reissner-Mindlin-Timoshenko plate-beam vibration model. IMA J. Appl. Math. 74:149-162. 